\title{
Enhanced supportive care is broader than palliative care
}

The title of this Letter by Berman and Davies (BMJ

2019;365:11629, doi:10.1136/bmj.11629) is wrong. It should be

"Supportive care is broader than palliative care." 